Meta

Journal des traducteurs

Translators' Journal

\title{
La terminologie de la neurolinguistique : perspectives diachroniques
}

\section{Terence MacNamee}

Volume 29, numéro 1, mars 1984

Cerveau, langage et traduction

URI : https://id.erudit.org/iderudit/003532ar

DOI : https://doi.org/10.7202/003532ar

Aller au sommaire du numéro

Éditeur(s)

Les Presses de l'Université de Montréal

ISSN

0026-0452 (imprimé)

1492-1421 (numérique)

Découvrir la revue

Citer cet article

MacNamee, T. (1984). La terminologie de la neurolinguistique : perspectives diachroniques. Meta, 29(1), 91-98. https://doi.org/10.7202/003532ar d'utilisation que vous pouvez consulter en ligne.

https://apropos.erudit.org/fr/usagers/politique-dutilisation/ 


\section{LA TERMINOLOGIE DE LA NEUROLINGUISTIQUE : PERSPECTIVES DIACHRONIQUES}

TERENCE MACNAMEE

\section{LES ENSEMBLES DISCURSIFS}

La "neuro-linguistique ", pourquoi pas ? On finit par s'accoutumer à ces approches multidisciplinaires au langage. Née de la coopération des aphasiologues et des linguistes de type scientifique, la discipline a produit déjà une littérature importante, et cela en plusieurs langues de recherche : anglais, français, allemand, russe. Interdisciplinaire, multilingue, battant les frontières de la science, la neurolinguistique révèle paradoxalement au traducteur, au terminologue un vocabulaire parfois traditionnel. Plusieurs termes remontent à des époques et à des auteurs assez lointains, où ils ont eu une signification moins précise ou plus concrète qu'aujourd'hui. On note également que le commentaire des savants sur la terminologie même est constant. Pas de domaine où l'inexactitude où l'imprécision des termes est plus vigoureusement condamnée! Toutefois, la terminologie de la neurolinguistique reste complexe et difficile, et le traducteur professionnel à qui l'on a confié un texte savant dans le domaine, les actes d'un congrès, ou le terminologue chargé d'assurer une meilleure compréhension internationale parmi les savants et les équipes de recherche, s'en rendent assez vite compte. Voyons la chose de plus près.

On constate d'emblée que la discipline désignée par le vocable de "neurolinguistique " est constituée par deux discours, deux composantes hétérogènes : neurologie et linguistique, c'est-à-dire, le discours anatomo-clinique sur le langage, et le discours phonétique-grammatical. Elle comporte donc deux grandes strates de vocabulaire : d'une part, l'anatomie et la physiologie du cerveau, et ses troubles (nosologie ou séméiologie), de l'autre, l'analyse de la structure du langage même. Le terme "neurolinguistique " est assez récent, bien entendu : il date des années 60 . On dirait donc que la discipline constituée par cette intersection de deux ensembles discursifs est presque nouvelle. Mais il n'en est pas ainsi. La tradition médicale, mais aussi la réflexion psychologique et philosophique sur "les causes physiques de l'éloquence" - c'est la formule de Cordemoy $(1668: 241)$ - remontent à l'Antiquité. Si l'on définit la neurolinguistique étroitement comme " la grammaire des aphasies " ou plus largement comme l'étude du support somatique du langage, le problème de la terminologie reste central. De Willis et Stenon à Broca, en passant par Gall, et en avançant jusqu'à Charcot et Freud, les grands dans le domaine se sont posé sans cesse la question du choix des mots pour saisir les phénomènes. La provenance des mots de deux ensembles discursifs différents n'explique pas complètement cet embarras des esprits.

Comment se fait-il alors que les progrès du discours sur le langage et le cerveau ont été tellement jalonnés par des débats terminologiques? En effet, des réformes de nomenclature ont accompagné toutes les avances dans le domaine. À vrai dire, c'est toute 
une tradition critique qui se dégage, un regard qui constate la confusion inhérente à des situations où le mot désigne une chose inexistante, ou fait prendre une partie pour le tout, ou le tout pour une partie, ou que la chose n'est pas désignée par un mot unique. La raison de cette confusion? Broca la trouvera plus tard dans l'attitude qui voyait dans l'anatomie cérébrale quelque chose de fantaisiste ou d'arbitraire, sans système, d'où " il était naturel qu'on n'éprouvât pas le besoin de déterminer le sens des mots dont on se servait pour en parler" $(1878: 193)$. Toutefois, dans les siècles précédents, le souci de réforme d'une terminologie inadéquate est resté constant, et Broca semble être peu renseigné sur quelques augustes devanciers : notamment Sténon (1669).

\section{LES RÉFORMATEURS}

Il faut comprendre l'état des connaissances sur le cerveau au XvIIre siècle. Face à l'héritage du monde antique, l'anatomie d'Hippocrate et de Galien, et aux innovations anatomiques de la Renaissance, notamment celles de Vésale (1545), l'Âge classique cherchait de nouveau les mécanismes du savoir et du discours dans le cerveau de l'homme. Des théories spéculatives se présentaient : pour le médecin anglais Th. Willis (1664), les grandes divisions de la masse cérébrale correspondaient aux diverses fonctions de l'esprit humain. Selon cette tentative de localisation des "facultés de l'âme " (et il s'agit bien sûr d'une conception des facultés tributaire de la tradition psychologique antico-médiévale) dans l'espace cérébral, ces facultés siègent dans le cerveau proprement dit, les corps striés servant à la perception des idées, le corps calleux à l'imagination et l'ensemble du cortex à la mémoire, tandis que les fonctions moins relevées, contrôle des viscères et des appétits, siègent dans le cervelet. Au contraire, Descartes, dans son Traité de l'Homme (1661), passe à côté du problème de la localisation des facultés pour loger l'esprit (ou tout au moins son lieu d'action physique) dans la glande pinéale. Il revint alors à Sténon, jeune chercheur scandinave déjà reconnu dans les milieux savants à Paris, de prendre ces théories spéculatives en exemple et de montrer comment la volonté de savoir préjuge et confond la connaissance de l'entité physique qu'est enfin le cerveau de l'homme.

«Il est encore arrivé, que ceux qui ont entrepris de faire ces explications, par je ne sçay quel esprit, qui s'est rencontré en la plus-part de ceux qui ont écrit des arts, ont employé des termes fort obscurs, des métaphores, \& des comparaisons si peu propres, qu'elles embarassent presque également l'esprit de ceux qui entendent la matière, et ceux qui s'en veulent instruire " $(1669: 27$ sq.). Sténon s'en prend tout d'abord à la bassesse et au mauvais goût de bien des termes dans l'anatomie cérébrale, "indignes de la partie matérielle de l'homme la plus noble ". En effet, la tradition médicale avait projeté dans le cerveau des ressemblances fugitives à d'autres parties du corps. "Quelle nécessité y avoit-il, se demande Sténon, d'employer les mots de nates, de testes, d'anus, de vulva, de penis, puisqu'ils ont si peu de rapport aux parties, qu'ils signifient dans l'Anatomie du cerveau? en effet, ils leur ressemblent si peu, que ce que l'un appelle nates, l'autre appelle testes, \&c. " Donc, pour Sténon, c'est une indignité de vouloir trouver des fesses ou des testicules dans le cerveau, mais encore, ces dénominations sont arbitraires et prêtent à la confusion.

Pour une raison analogue, Sténon critique le terme de troisième ventricule comme "fort équivoque " (1669:29 sqq.). Pour les Anciens, c'était " une cavité sous la fornix, ou la voûte, laquelle voûte ils croyoient séparée de la base du cerveau ", alors que pour Sylvius, contemporain de Sténon, c'est « un canal qui se trouve dans la substance de la base du cerveau, entre l'entonnoir, et le passage qui va sous les deux pairs postérieurs des tubercules du cerveau, vers le quatrième ventricule ». Il précise en outre que certains anatomistes " en disséquant, séparent les corps du deuxième pair de tubercules, et pren- 
nent pour le troisième ventricule, l'espace entier qui se trouve entre ces deux corps, ce qu'ils ont fait en les séparant ". Donc, trois significations possibles pour le terme de troisième ventricule :

$1^{\circ}$ "la fente qui est au-dessus "

$2^{\circ}$ "le canal de dessous"

$3^{\circ}$ "l'espace d'entre le canal et la fente"

Seule la signification (2) est "vraye dans le naturel ". Les deux autres " dépendent entièrement de la préparation $"$.

Autre exemple : le corps calleux (pris dans une acception plus large au XVII ${ }^{e}$ siècle qu'aujourd'hui) « signifie la substance blanche du cerveau, qu'on void quand on en sépare les deux parties latérales ; mais il est vray que cette partie est entièrement semblable au reste de la substance blanche du cerveau ; \& ainsi l'on ne void point de raison, de donner un nom particulier, à une partie de cette substance " (1669:31).

Il s'agit donc chez Sténon d'une critique poussée de signifiants qui désignent mal leur signifié ou dont le signifié est inexistant ou mal défini. Nous ne restituerons toutefois à cette critique la plénitude de son sens que si nous y voyons le contre-courant de la tendance classique de projeter des modèles spéculatifs de fonctionnement sur l'énigme cérébrale. En effet, le cerveau se montrait toujours rebelle à l'analyse structurale. Comme le dit Sténon lui-même, "vous voyez sur la surface, des diversités qui méritent l'admiration; mais quand vous venez à pénétrer jusqu'au dedans, vous n'y voyez goutte... " (1669: 3). L'absence de repères anatomiques, la pléthore de termes souvent métaphoriques, risquaient sans cesse de fausser les observations et d'égarer le raisonnement.

Première grande tentative de poser une terminologie après Sténon : Gall. Le fondateur de la phrénologie fut un grand anatomiste, un esprit révolutionnaire de la fin du XVIII ${ }^{\mathrm{e}}$ siècle. À Gall revient le crédit d'avoir présenté une méthode moderne de disséquer le cerveau humain et donc de connaître sa structure essentielle. Son grand rival Flourens dit : “Je n'oublierai jamais l'impression que j'éprouvais la première fois que je vis Gall disséquer un cerveau. Il me semblait que je n'avais pas encore vu cet organe " (1843 : 186). Mais les innovations terminologiques du mouvement phrénologique visaient les fonctions qu'il s'agissait de localiser dans l'espace cérébral plutôt que le détail structural ou morphologique. Ainsi Gall lui-même distinguait 27 "facultés et penchants "susceptibles de localisation dans le cerveau de l'homme, son collaborateur Spurzheim 35.

Gall, grand anatomiste qu'il était, regardait le cerveau seulement comme un objet médiat de science, son vrai but étant de fonder une discipline certaine sur le caractère et le comportement de l'homme. Nous voyons ici, dans ses débuts, l'entreprise savante de tout le siècle qui va suivre (et du nôtre, bien entendu), celle de dresser une classification évaluative des individus, de leurs aptitudes et de leurs défauts, à partir d'un ensemble de normes biologiques et constitutionnelles. Là ou les "localisateurs" du XVIII siècle comme Ch. Bonnet s'étaient contentés d'adopter " des fonctions du cerveau déduites de la classification traditionnelle des sens, en leur proposant des lieux en rapport à la fois avec le trajet et le rôle présumé de certaines voies périphériques", comme le note $G$. Lanteri-Laura (1970: 56), l'innovation majeure du mouvement phrénologique fut l'élaboration d'un système de nomenclature de tous les aspects de la vie psychique de l'homme. Entreprise purement chimérique? À l'époque, les opinions étaient partagées. L'essentiel de la méthode de Gall consiste en l'identification d'aires corticales diffuses sur la surface du cerveau ayant des fonctions de pensée ou de conduite, l'hypothèse directrice étant que la prééminence de ou telle faculté ou penchant dans un individu se montrera par l'hypertrophie de l'" organe cérébral "correspondant, et, en outre, que 
cette hypertrophie cérébrale se traduira par une prééminence des os du crâne dans ce même endroit. C'est pour cette raison que Gall et ses disciples palpent les bosses du crâne des vivants pour connaître leur caractère, leurs aptitudes, comme ils dissèquent le cerveau post mortem. Ce faisant, Gall crée toute une nomenclature des fonctions humaines, supérieures et inférieures : "instinct de la propagation " (qui siège au cervelet, hypertrophié bien sûr chez les érotomanes), " mémoire verbale » (qui siège à la moitié inférieure des lobes frontaux, et qui fait que ceux qui ont le don des langues ont des yeux exorbités), et «sens du langage et de la parole » (à la partie moyenne de la face orbitaire des lobes frontaux). Si toute cette systématisation nous semble aujourd'hui dérisoire, l'élaboration d'une nomenclature fonctionnelle n'est pas restée sans conséquences. En effet, l'orientation du discours savant vers le comportement et ses racines cachées dans le cerveau a rendu possible tout le débat moderne sur la localisation des fonctions, enfin l'intersection des deux ensembles discursifs que nous avons évoqués au départ ; puisque ce débat, à partir des affirmations de Gall et des controverses qui les ont suivies, se trouve centré surtout sur le langage et son support cérébral.

C'est ainsi que Bouillaud (1825), tout en niant par exemple le rôle privilégié du cervelet dans la sexualité que lui avait conféré Gall, a pu insister sur " le principe de la pluralité des organes cérébraux ", surtout en ce qui concerne le langage. Gall, pour fonder sa localisation du sens de la parole, s'était penché sur des cas où les atteintes bilatérales de cette région entrainent, chez l'enfant, une impossibilité isolée d'apprendre à parler en contraste avec un développement normal de l'intelligence, et, chez l'adulte, des troubles divers du langage et parfois un mutisme : ces troubles, comme le note $G$. Lanteri-Laura (1970:110), deviennent très vagues et ne sont presque pas décrits pour eux-mêmes, mais la référence à la pathologie est fondamentale. Bouillaud, pour sa part, se demande (1825: 11) : "en rejetant (la doctrine de la pluralité des organes cérébraux), comment se rendre raison, par exemple, du bégaiement, de la mutité chez l'homme, de l'absence de la parole chez tous les animaux, phénomène coïncidant avec toute la plénitude et toute la perfection des autres fonctions musculaires? "Il tranche la question en posant dans le cerveau un organe du langage articulé, qui exécute la parole, et également un organe de " la partie intellectuelle de la parole ", ou "parole intérieure ». Bouillaud se penche sur des cas de perte de l'articulation avec conservation de l'intelligence, et il localise ce principe exécutif de la parole, à titre provisoire, dans "les lobules antérieurs ". Ce sera, bien entendu, plus tard, "la faculté du langage articulé " de Broca (1861), qui sera localisée, non plus dans un champ cortical diffus, mais dans une entité structurale circonscrite du cortex : la troisième circonvolution frontale gauche.

Donc, la nouvelle nomenclature des fonctions introduite par Gall et ses disciples, bien qu'elle fût tirée du langage commun et de l'expérience anecdotique plutôt que de la science, et attaquée par ses adversaires comme arbitraire ou fantaisiste (" Gall et Spurzheim parlent beaucoup de nomenclature, dit Flourens dans son Examen de la Phrénologie (1843 : 86), mais ils ne voient pas qu'en matière de nomenclature, la première difficulté, et la seule, est d'arriver aux faits simples. Qui est arrivé aux faits simples, a bientôt une bonne nomenclature. »), cette nouvelle nomenclature fonctionnelle a été indispensable pour l'exploration, la cartographie des bases cérébrales du comportement. C'est elle, en effet, qui a permis finalement l'élaboration des grands schémas de centres (on parlera bientôt de "centres » corticaux) qui ont dominé la conception du fonctionnement cérébral chez les grands neurologistes du XIX ${ }^{e}$ siècle.

Comme nous l'avons déjà constaté à plusieurs reprises, les progrès dans le domaine du support cérébral du langage s'accompagnent toujours de réformes terminologiques. L'époque « moderne " de l'aphasiologie s'inaugure de façon décisive dans les travaux cliniques de Broca, et nous avons de lui en même temps un essai de terminologie 
(1878). Selon Broca, la nomenclature du cerveau avant le XIX ${ }^{\mathrm{e}}$ siècle n'était pas fixée, parce qu'on n'avait pas encore l'idée d'une anatomie cérébrale constante. Ainsi Broca dans son enquête vise surtout l'anatomie du cortex, donnant des définitions précises notamment aux termes lobe, circonvolution, pli, écorce, et il prend comme sous-titre de son article " dénomination des divisions et subdivisions des hémisphères et des anfractuosités de leur surface ". En effet, l'attitude critique de Broca à l'égard de ses prédécesseurs est justifiée, dans la mesure que l'époque classique n'accordait pas de statut privilégié au cortex, même dans ses tentatives de localisation fonctionnelle. Comme le souligne Broca dans son exposition, le mot même de circonvolution est emprunté, à l'origine, à la description de l'intestin. Les auteurs d'autrefois, ne voyant ni structure ni régularité sur la surface corticale, négligeaient de lui appliquer une nomenclature précise. Dans le mouvement de réforme qu'il veut lancer, Broca se donne à peu près le même but que Sténon : "que chaque chose ait un nom, qu'elle n'ait qu'un seul, et que ce nom ne désigne qu'une seule chose " (1878: 197). Comme Sténon, également, il constate que l'embarras des esprits à l'égard de la nomenclature cérébrale vient aussi bien de la difficulté constante d'identifier les choses (c'est-à-dire, les éléments de structure anatomique et topographique) que de la difficulté de choisir les mots justes pour les désigner.

"Je ne me propose pas de renouveler toute la nomenclature cérébrale, dit Broca un peu plus loin (1878:205), je me borne à chercher la précision du langage. »C'est à propos du terme circonvolution justement qu'il parle, le trouvant trop long (les Anglais disent "convolution"; et pourquoi pas tout simplement "volution"?) et peu exact (" circum " suggère l'idée d'un enroulement autour d'un centre), mais il finit par le garder puisque consacré par l'usage professionnel.

La critique de Broca diffère essentiellement dans sa portée et ses ambitions des grands efforts de fonder des terminologies et des classifications scientifiques qui ont marqué le Siècle des Lumières. La grande systématisation des êtres vivants accomplie par Linné, par exemple, ou celle pratiquée en chimie par Lavoisier, sont restées sans analogue dans le domaine du cerveau. Tout ce qui intéresse un réformateur comme Broca, ou comme Sténon au XVII siècle, c'est que le rapport des choses et des noms qui les distinguent soit unique et constant ; "il n'est nullement nécessaire, dit Broca (1878 : 196), que ces noms soient nouveaux et assujettis à certaines règles de dérivation et de composition. " C'est que, face au cerveau humain, l'on garde toujours le sentiment d'être devant un inconnu, ou tout au moins un objet peu à peu à explorer, une terre vierge qui garde encore ses secrets. La nomenclature anatomo-clinique et fonctionnelle du cerveau ne pouvait être un système clos, une totalité, puisque la connaissance du cerveau et de ses fonctions ne l'était pas.

Cette qualité provisoire de la terminologie de notre discipline s'explique donc en grande partie par l'incertitude, l'impermanence réfléchie des connaissances. Ainsi la terminologie dont nous sommes aujourd'hui les héritiers reste hétérogène, et maint terme curieux rappelle une théorie désuète. Est-il besoin d'évoquer à ce sujet le terme réflexe, issu de l'homme mécanique de Descartes (1661), fondé à son tour sur des notions optiques?

C'est le XIX ${ }^{e}$ siècle, avec Broca entre autres, qui a dégagé la notion capitale d'une diachronie de la terminologie. Pour ce XIXe siècle qui avait pu démontrer le principe du changement historique à la base du langage humain, il était évident que la terminologie savante est chose instable ; contraire à la conception condillacienne d'une image "langue bien faite". Ainsi Cl. Bernard, oeuvrant, en même temps que Broca, à l'élaboration d'une médecine scientifique moderne, précise que les mutations diachroniques du langage de la science apportent souvent des erreurs et des malentendus (1865:262) : 
Quand on crée un mot pour caractériser un phénomène, on s'entend en général à ce moment sur l'idée qu'on veut lui faire exprimer et sur la signification exacte qu'on lui donne, mais plus tard, par les progrès de la science, le sens du mot change pour les uns, tandis que pour les autres le mot reste dans le langage avec sa signification primitive. Il en résulte alors une discordance qui, souvent, est telle que les hommes, en employant le même mot, expriment des idées très différentes. Notre langage n'est en effet qu'approximatif, et il est si peu précis, même dans les sciences, que, si l'on perd les phénomènes de vue pour s'attacher aux mots, on est bien vite en dehors de la réalité. On ne peut alors que nuire à la science quand on discute pour conserver un mot qui n'est plus qu'une cause d'erreur, en ce sens qu'il n'exprime plus la même idée pour tous.

C'est dire que les progrès du discours savant provoquent un glissement de sens dans les termes de ce même discours. $\mathrm{Cl}$. Bernard appartient donc à la tradition critique, représentée également par Broca, qui montre sans cesse le danger de se laisser égarer par les mots du discours et de ne plus raisonner sur les choses. C'est un commentaire en marge du discours savant qui le critique sans toutefois le supplanter, puisque malgré tout le langage est "un organisme qui croit sans cesse, et sans cesse rejette des parties mortes ", comme dit Brunot ( $H$. L. fr. VI, 1, 2:665). Mais cette perspective critique sur la terminologie savante, son arbitraire, sa qualité essentiellement instable et ouverte, n'explique pas tout à fait l'inertie des couches historiques de termes qui forment le discours de notre discipline. D'abord il faut projeter les termes sur un fond plus large, il faut dégager un moyen terme entre les mots et les choses.

\section{LES LIEUX COMMUNS}

Ce qui importe, historiquement, pour comprendre l'évolution des connaissances sur le cerveau et son fonctionnement, ce n'est pas seulement la qualité toujours partielle du savoir, mais aussi la volonté de combler les lacunes par le moyen de ce qu'on croit voir ; saisissant tant bien que mal la nature du cerveau tout en lui imposant un modèle.

Dans une étude des plus intéressantes, Magoun (1958 : Introduction) a présenté l'histoire des notions sur le cerveau comme une série de modèles interprétatifs empruntés au contexte historique de chaque époque. Ainsi la médecine gréco-romaine et ses prolongements médiévaux et humanistes a mis en place un modèle hydraulique dérivé du génie civil et militaire de l'Antiquité, par exemple, les aqueducs et les ponts, et qui dirige son attention surtout aux ventricules. Au XVII ${ }^{e}$ siècle, avec le Traité de l'Homme de Descartes et la pensée mécaniste, apparaît un modèle dioptrique, fondé sur les expériences touchant la lumière, la vue et les prismes, et qui nous a donné le terme de réflexe. Un modèle phrénologique lui a succédé, toujours selon le classement de Magoun, au tournant du XVIIIe siècle, et alors commencent les grands modèles historiques du XIXe siècle : un modèle géologique où le cerveau ressemble à la terre non seulement dans sa forme de globe mais aussi en ce qu'il révèle une série de strates horizontales superposées l'une sur l'autre dans le temps évolutif $(1958: 12)$, et un modèle embryologique, où le cerveau est constitué à partir d'éléments sous-corticaux des plus primitifs. Enfin, à notre ère, voilà les modèles technologiques : pour nous, le cerveau est un central téléphonique, ou un ordinateur. (Et comment le concevoir autrement, si ces entités s'imposent sans cesse à notre pensée, à nos perceptions?)

Ce sont là des métaphores à la deuxième puissance pour ainsi dire, des lieux communs générateurs de termes, qui forment et contraignent notre discours. Si nous avons tendance à parler de nos cerveaux en termes d'informatique ou de gestion de l'information, c'est que notre langage trahit la vection de notre pensée tout en annonçant sa finitude. Un modèle interprétatif du cerveau et de son fonctionnement rend possible l'explication, en même temps qu'il lui impose des limites. Et puisque ces modèles sont toujours 
mi-conscients et primaires dans la perception, leurs influences ont été tenaces dans la terminologie. Le terme survit au modèle, signalant toutefois la couche historique où il appartient. Cela dit, nous avons toujours à motiver, à expliquer cette richesse métaphorique, cette prodigalité du discours à l'égard du cerveau et du langage au fil des siècles.

\section{LES MÉCANISMES DE PROJECTION}

Nous avons insisté sur le caractèere double de la terminologie neurolinguistique, issue de l'intersection de deux ensembles discursifs. Cette terminologie "à deux faces" est le reflet d'une grande question épistémologique : nous disons que le langage "se trouve dans $»$ le cerveau... mais comment exactement ? Même question que se posaient les contemporains de Gall ; même question que se posent les successeurs de Broca, une fois mis en doute le dogme de la troisième circonvolution frontale gauche. Si nous projetons le langage dans le cerveau pour ainsi dire, c'est que les hommes ont toujours projeté leurs préoccupations, leurs conceptions de la vie mentale sur cet organe visiblement inerte. L'apothéose cartésienne de la glande pinéale, le bilan des fonctions psychiques attribuées à la substance grise et à la substance blanche, de Willis à Bouillaud, enfin le phrénologiste palpant les bosses du crâne, tout parle d'une volonté acharnée de tirer les clés du savoir de ce cerveau, régi par la Lune (selon les médecins-astrologues de la Renaissance) et froid comme elle. Si le cerveau est un sensorium ou entrepôt des données de l'expérience vécue, tout y est projeté, comme les parties du corps qui déplaisaient tellement à Sténon, ou la "réflexion" du stimulus chez Descartes, ou alors les perceptions d'objets emmagasinées selon Willis dans les cellulae aut apothecae de la masse cérébrale, comme "les traces de mémoire" ou les "engrammes" des conceptions modernes.

Mais les projections vont plus loin, comme le montre la localisation du langage et d'un univers de fonctions supérieures dans le cerveau, par Gall, par Bouillaud, par Broca. C'est l'unique siège du mystère humain, une fois chassé le cardiocentrisme de la tradition péripatéticienne : Harvey, pour qui le coeur est encore "le Soleil du Microcosme ", principe de vie et d'action, coupe résolument ses racines mythiques en démontrant sa vraie fonction dans la circulation du sang, et c'est Sténon lui-même qui achève le processus en déclarant cor vere musculus est, c'est-à-dire, un mécanisme corporel entre autres (1664). Le cerveau devient alors la dernière citadelle du secret, et le siège commence.

Pour les hommes de l'époque classique, l'anatomie cérébrale était d'abord un voyage de découverte, la conquête d'un nouveau monde. En premier lieu, la saisie de quelques traits saillants du paysage, quelques points de repère, sans pouvoir nécessairement deviner leur rôle dans le tout ; comme sur les cartes de la Nouvelle France où l'on voit entourée d'espace blanc une "rivière dont on ne connoît ni la source ni l'embouchure ". À vrai dire, le cerveau se prêtait mal à l'attention des anatomistes, et en le disséquant l'on se trouvait bien déçu. Â rappeler les mots de Sténon (1669:3), « vous voyez sur la surface des diversités qui méritent l'admiration ; mais quand vous venez à pénétrer jusqu'au dedans, vous n'y voyez goutte »: quelques arpents de neige.

La nomenclature, c'est l'imposition d'une géographie. (Hume, pour sa part, parlait de "géographie mentale "; lui qui n'était certainement pas neurologiste avait déjà l'idée, comme Gall, que les aptitudes, les capacités humaines pouvaient occuper un espace ; un espace qui allait bientôt se concrétiser dans le cortex...) Aujourd'hui, le terroir est bel et bien conquis, l'état de siège se prolonge.

Mais toujours un sentiment furtif : C'est trop tard. Comme la conquête de la lune, c'est l'escale inutile, sur une planète refroidie où rien ne bouge. Ramasser quelques pierres, voilà le butin de la curiosité. Nous ne voulons pas voir que le discours le plus élo- 
quent finit en silence, que sous les structures de structures, au terme de ce long mouvement centripète vers l'essence humaine, il n'y a peut-être ... Rien.

Les hommes d'autrefois voyaient dans le monde un grand Livre ouvert à qui sait lire. "Après que j'eus employé quelques années à étudier ainsi dans le livre du monde et à tâcher d'acquérir quelque expérience, je pris un jour la résolution d'étudier aussi en moi-même", dit Descartes (Discours 1). Le corps, le fonctionnement humain, était lui aussi un texte à déchiffrer. Aujourd'hui, le cerveau de l'homme, la base cérébrale de son langage, nous apparaît peut-être comme un livre trop connu où nous voyons ce que nous voulons lire.

Dans les pages précédentes de cet essai, nous avons pu évoquer assez sommairement quelques moments de la "préhistoire" de notre discipline qui nous ont paru importants dans l'élaboration de sa terminologie. La neurolinguistique est appelée de nos jours à fournir de nouveaux modèles, à concevoir peut-être d'une autre manière le fonctionnement du langage dans le système nerveux central. La réflexion, toutefois, reste tributaire des mots qu'elle emploie pour s'exprimer, sinon pour saisir l'impénétrable. Dans ces circonstances, le modèle passe pour une sagesse, et la métaphore devient réalité. C'est sans doute à cause du caractère constant de l'énigme cérébrale que les grands de la discipline, de Sténon, à Gall, à Broca, ont toujours porté une attention critique aux mots d'un discours riche et incertain.

\section{BIBLIOGRAPHIE}

BERNARD, Cl. (1865) : Introduction à l'étude de la médecine expérimenale, Paris, Garnier-Flammarion. BOUILLAUD, J.-B. (1825) : Archives générales de médecine, VIII, pp. 25-45.

BROCA, P. (1878) : "Nomenclature cérébrale : dénomination des divisions et subdivisions des hémisphères et des anfractuosités de leur surface ", Revue d'anthropologie, 2e série, t. I, pp. 193-236.

BRUNOT, F. (1966) : Histoire de la langue française, nouvelle édition, Paris, A. Collin.

CLARKE, E. et C.D. O'MALLEYA (1968) : The Human Brain and Spinal Cord, Berkeley, University of California Press.

CORDEMOY, G. de (1668) : Discours physique de la parole, Paris.

DESCARTES, R. (1661) : Traité de l'Homme, Leyde.

FLOURENS, P. (1843) : Examen de la phrénologie, Paris, Paulin.

GALL, F.J. et G. SPURZHEIM (1819) : Anatomie et physiologie du sysième nerveux en général et du cerveau en particulier, Paris, 4 vol.

LANTERI-LAURA, G. (1970) : Histoire de la phrénologie : L'homme et son cerveau selon F.J. Gall, Paris, PUF.

MAGOUN, H.W. (1958) : The Waking Brain, Springfield, Ill., Thomas.

STÉNON, N. (1664) : De Musculis et Glandulis, Amsterdam.

STÉNON, N. (1669) : Discours sur l'anatomie du cerveau, Paris.

VÉSALE, A. (1545) : De corporis humani fabrica, Basileae.

WILLIS, Th. (1664) : Cerebri anatome, Londres. 